\title{
Observation of interdot coupling phenomena in nanocrystalline silicon point-contact structures
}

\author{
M.A.H. Khalafalla ${ }^{\text {a,c,* }}$, H. Mizuta ${ }^{\text {a,c }}$, S. Oda ${ }^{a, c}$, Z.A.K. Durrani ${ }^{\text {b,c }}$ \\ ${ }^{a}$ Quantum Nanoelectronics Research Centre, Tokyo Institute of Technology, O-Okayama, Meguro-ku, Tokyo 152-8552, Japan \\ ${ }^{\mathrm{b}}$ Department of Engineering, University of Cambridge, Trumpington Street, Cambridge CB2 1PZ, UK \\ c SORST JST, Japan Science and Technology, 4-1-8, Honcho, Kawaguchi-shi, Saitama 332-0012, Japan
}

Available online 27 December 2005

\begin{abstract}
We have investigated the electrostatic and coherent couplings in $30 \mathrm{~nm} \times 30 \mathrm{~nm} \times 40 \mathrm{~nm}$ nanocrystalline silicon side gated point-contact transistors where the nanocrystalline silicon grains (10-35 $\mathrm{nm}$ in size) formed naturally coupled quantum dots through the thin grain boundary layers. Measurement at $4.2 \mathrm{~K}$ of the current as a function of the gate voltages in some thermally oxidised and high temperature annealed devices showed switching of the Coulomb oscillation peaks that was associated with electrostatic coupling between two major nanocrystals embedded in the point-contact channel. We also observed in similar measurements, using an oxidised-only device, an enhanced switching of the peaks with additional peak structures in the switching region where the grains coupled strongly. The characteristics in this region were well fitted to a sum of four Lorentzian peaks. These peaks were associated with coherent tunnelling of the electrons through the delocalised electron wavefunctions over the strongly coupled grains across the low and thin grain boundary tunnel barriers.
\end{abstract}

(c) 2005 Elsevier B.V. All rights reserved.

PACS: $73.23 . \mathrm{Hk} ;$ 73.63.Bd; 78.67.Hc

Keywords: Nanocrystalline silicon; Electrostatic coupling; Coherent coupling

As-deposited nanocrystalline silicon (nc-Si) films consist of nanometer scale crystalline silicon grains separated by amorphous silicon grain boundary layers. Chemical Vapour Deposition (CVD) techniques can be used to easily deposit nc-Si films with small size grains (e.g. $8 \mathrm{~nm}$, [1]) over large area substrates. With the possibility of modifying and controlling the height of potentials at the grain boundaries, using for example thermal processes such as oxidation and annealing [2], the electrons in the grains can be confined to quantised levels. Therefore the grains may form naturally grown artificial atoms (quantum dots, QDs) with large confinement energies raising the possibility

\footnotetext{
${ }^{*}$ Corresponding author. Address: Quantum Nanoelectronics Research Centre, Tokyo Institute of Technology, O-Okayama, Meguro-ku, Tokyo 152-8552, Japan. Fax: +81 357342542.

E-mail address: khalafalla@neo.pe.titech.ac.jp (M.A.H. Khalafalla).
}

of observation of room temperature single electron charging effects in nc-Si systems [3]. The densely packed nc-Si QDs can couple electrostatically to form artificial ionic molecule systems, or coherently to form artificial valence molecule systems [4]. Investigation of these systems may be important for future quantum information technology [5]. Furthermore, silicon based quantum information systems are compatible with the current advances in the VLSI technology [6].

In electrostatically coupled QDs a change in the electron number of a QD by one can induce a charge on the other QD changing its electrostatic potential. Transport spectroscopy can be used to investigate electrostatic coupling in the double QDs systems [7]. Here a charge stability diagram is formed by the Coulomb oscillation peak lines as a function of the gate voltages where the peak lines trace a pattern of hexagonal regions of charge stability in the double QDs. 
The charges between neighbouring hexagonal regions changes by one electron and three adjacent hexagonal regions meet at a triple point where the electrochemical potentials of the QDs are aligned. In the vicinity of two adjacent triple points, the QDs are strongly coupled with coupling energy measured by the splitting of the Coulomb oscillation peaks in this region. If the QDs are coherently coupled through a coupling barrier much thinner than the electron inelastic mean free path, an electron can oscillate between these QDs without inelastic scattering. This causes the electrochemical potentials of the QDs near a triple point to split into two delocalised levels over the QDs. Thus, in the vicinity of two triple points, four levels will be available for the electron transport across the coherently coupled QDs. This enhances the splitting of the oscillation peaks in the strong coupling region by the tunnel splitting energy [4].

Most of the past studies on coupled QDs systems have used two-dimensional electron gas (2DEG) QDs in GaAsAlGaAs heterostructures [4]. Only few reports have investigated coupled QDs in silicon [8,9]. The sizes of the fabricated QDs in these investigations were limited by the resolution of the electron beam lithography to about $30 \mathrm{~nm}$ which in turn limits the observation of coupling effects to milli-kelvin temperatures.

This paper investigates the effect of electrostatic and coherent coupling in the electron transport through a few coupled nc-Si QDs embedded in $30 \mathrm{~nm} \times 30 \mathrm{~nm} \times 40 \mathrm{~nm}$ nc-Si point-contact channel (Fig. 1(a)) at a temperature of $4.2 \mathrm{~K}$. The nc-Si QDs are separated by $\sim 1 \mathrm{~nm} \mathrm{SiOx}$ grain boundary suboxide and are $10-35 \mathrm{~nm}$ in size, far smaller than the 2DEG QDs [7]. Two in-plane side gates

(a)

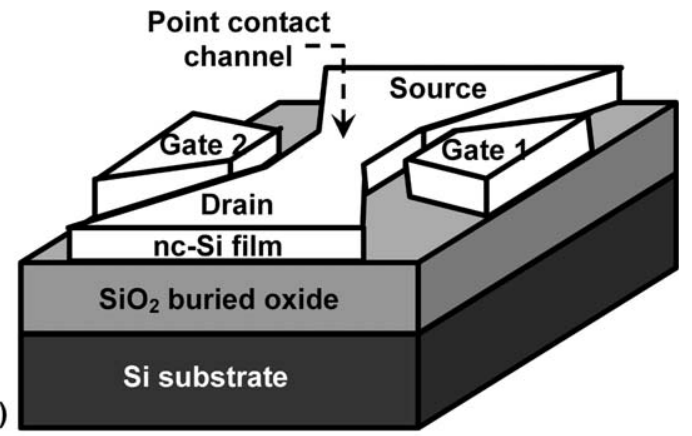

(b)

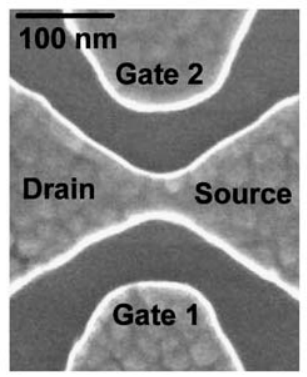

Fig. 1. (a) Schematic diagram of a nanocrystalline silicon point-contact transistor with two side gates. (b) Scanning electron micrograph of a nanocrystalline silicon point-contact transistor. have been used to modulate the electrostatic potentials in the nc-Si QDs. The stability charge diagrams are then formed by plotting the conductance/current as a function of the side gate voltages. Here we observed electrostatic coupling effects in some of the thermally processed (using combination of low temperature oxidation and high temperature annealing) devices and coherent coupling effects in an oxidised-only device.

The point-contact transistors were fabricated on $40 \mathrm{~nm}$ thick nc-Si film (n-type doped with Phosphorus at $1 \times 10^{19} \mathrm{~cm}^{-3}$ ) deposited by Low Pressure Chemical Vapour Deposition (LPCVD) at $700{ }^{\circ} \mathrm{C}$ on $150 \mathrm{~nm} \mathrm{SiO}_{2}$ buried oxide thermally grown on un-doped crystalline silicon substrate. Fig. 1(a) shows a schematic of the point-contact transistor with two side gates. High resolution electron beam lithography was used to fabricate the point-contact transistors on a PMMA resist layer on the nc-Si film, followed by reactive ion etching $\left(\mathrm{CF}_{4} / \mathrm{SiCl}_{4}\right.$ gases $)$ to trench isolate the devices and the gate electrodes. We then used different combination of low temperature oxidation, in $\mathrm{O}_{2}$ gas ambient, and high temperature annealing, in $\mathrm{Ar}$ gas ambient, to modify the grain boundary tunnel barriers [2]. Fig. 1(b) is a Scanning Electron Micrograph (SEM) of a completely fabricated device. This paper will demonstrate the measurement results in two devices, Device 1 which was oxidised at $750{ }^{\circ} \mathrm{C}$ for $60 \mathrm{~min}$ and annealed for $5 \mathrm{~min}$ at $1000{ }^{\circ} \mathrm{C}$, and Device 2 which was only oxidised for $30 \mathrm{~min}$ at $750^{\circ} \mathrm{C}$. The longer oxidation time and the annealing processes $[2,4]$ of Device 1 may raise its grain boundary potentials higher than the grain boundary potentials in Device 2. This may enhance the electrostatic coupling in Device 1, and the delocalisation of the electron wavefunctions in Device 2.

Fig. 2(a) shows the three-dimensional grey scale plot of the current $\left(I_{\mathrm{ds}}\right)$ in Device 1 as a function of the gate voltages, $V_{\mathrm{g} 1}$ and $V_{\mathrm{g} 2}$, at source-drain voltage $V_{\mathrm{ds}}=-6 \mathrm{mV}$ and a temperature of $4.2 \mathrm{~K}$. We observe a pattern of broadened Coulomb oscillation peaks (dark regions, max. $I_{\mathrm{ds}}=1.2 \mathrm{pA}$ ) and narrow oscillation valley regions (white regions, $I_{\mathrm{ds}} \approx 0 \mathrm{pA}$ ). The valleys shift linearly in the plane of $V_{\mathrm{g} 1}$ and $V_{\mathrm{g} 2}$ and break at some regions where the associated peaks switch to other adjacent peaks. For example, the valleys 'a' switch to 'b' (Fig. 2(a)) which may imply that the peaks associated with 'a' switch to the peaks associated with ' $b$ '. We associate the switching of the peaks with electrostatic coupling between two dominant grains in the point-contact channel.

We further investigate these switching effects using Monte Carlo single electron circuit simulation of models of two coupled QDs. These models are shown in Fig. 2(b). In Model ' $S$ ' the double QDs (QD1 and QD2) are coupled in series through the tunnel barrier ' $t$ ', QD1 and QD2 are capacitively coupled to $V_{\mathrm{g} 1}$ and to $V_{\mathrm{g} 2}$ via the cross and direct gate capacitances, QD1 is coupled to the source (grounded) via a tunnel barrier and QD2 is coupled to the drain (drain voltage, $V_{\mathrm{d}}$ ) via a tunnel barrier. Model ' $\mathrm{F}$ ' is formed by adding a tunnel barrier to Model 

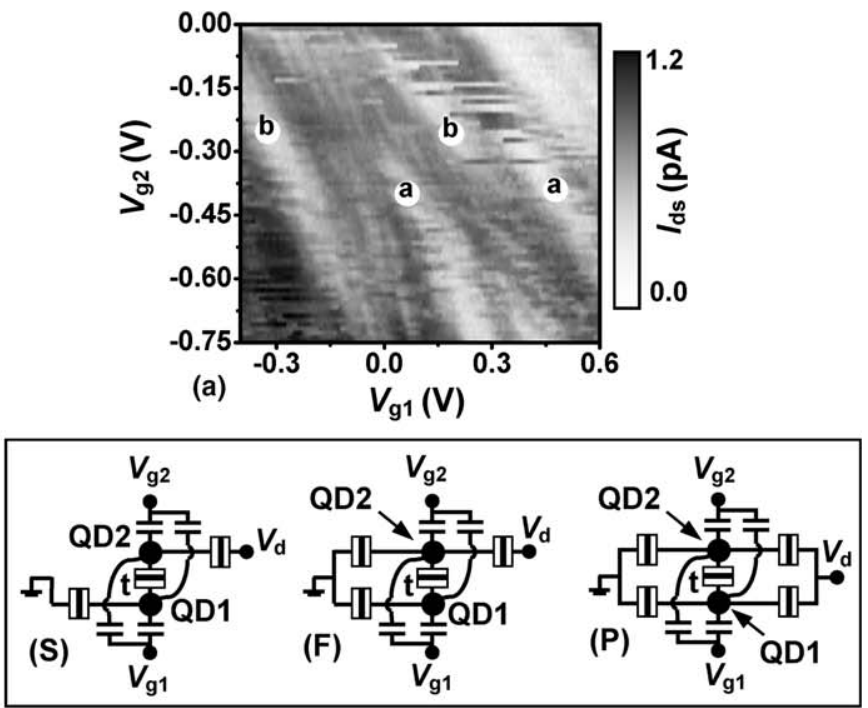

(b)

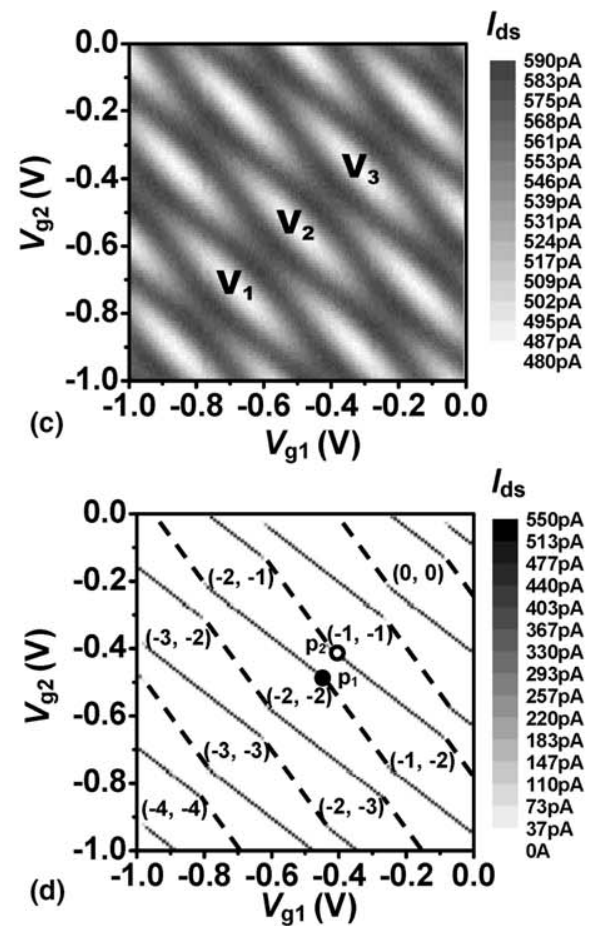

Fig. 2. (a) Experimental grey scale characteristics of the current, $I_{\mathrm{d}}$, in a point-contact transistor vs the gate voltages, $V_{\mathrm{g} 1}$ and $V_{\mathrm{g} 2}$, at source-drain bias $V_{\mathrm{ds}}=-6 \mathrm{mV}$ and $T=4.2 \mathrm{~K}$. (b) Models of two coupled quantum dots, QD1 and QD2, used in Monte Carlo simulations. (c) The Monte Carlo simulation of the current, $I_{\mathrm{ds}}$ vs $V_{\mathrm{g} 1}-V_{\mathrm{g} 2}$ at $4.2 \mathrm{~K}$ and at drain voltage $V_{\mathrm{d}}=2 \mathrm{mV}$ using Model ' $\mathrm{F}$ ' (see (b)) with tunnel junction capacitances of $30 \mathrm{aF}$, gate capacitances of $0.6 \mathrm{aF}$ and cross gate capacitances of $0.3 \mathrm{aF}$. (d) The Monte Carlo simulation of, $I_{\mathrm{ds}}$ vs $V_{\mathrm{g} 1}-$ $V_{\mathrm{g} 2}$ at $4.2 \mathrm{~K}$ and at $V_{\mathrm{d}}=2 \mathrm{mV}$ using Model 'F' (see (b)) with tunnel junction capacitances of $1 \mathrm{aF}$, gate capacitances of $0.6 \mathrm{aF}$ and cross gate capacitances of $0.3 \mathrm{aF}$.

' $\mathrm{S}$ ' coupling QD2 to the source. Model ' $\mathrm{P}$ ' is formed by connecting the floating QD1 in Model ' $\mathrm{F}$ ' to the drain via a tunnel barrier.

Fig. 2(c) shows the grey scale plot of the simulated $I_{\mathrm{ds}}$ vs $V_{\mathrm{g} 1}$ and $V_{\mathrm{g} 2}$ characteristics at $4.2 \mathrm{~K}$ and $V_{\mathrm{d}}=2 \mathrm{mV}$ using
Model ' $F$ '. Here we used QDs with small charging energies estimated as $e^{2} / C_{\mathrm{QD} 1}=2 \mathrm{meV}$ for $\mathrm{QD} 1$ and $e^{2} / C_{\mathrm{QD} 2}=$ $3 \mathrm{meV}$ for $\mathrm{QD} 2$ where $C_{\mathrm{QD} 1}$ and $C_{\mathrm{QD} 2}$ are respectively the total capacitances of QD1 and QD2 calculated by adding the tunnel barrier capacitances ( $30 \mathrm{aF}$ each) and the gate capacitances (cross: $0.3 \mathrm{aF}$ each, and direct: $0.6 \mathrm{aF}$ each) coupled to each QD. The characteristics show a pattern of broadened Coulomb oscillation peaks (dark regions) and narrow oscillation valleys (white regions) that are broken at some regions. This pattern is associated with electrostatic coupling between QD1 and QD2 with small charging energies and is consistent with the experiment (Fig. 2(a)).

Similar pattern can be obtained with Model ' $S$ ' and Model 'P' using QDs with average charging energies of $2 \mathrm{meV}$. The broadening effects in the Coulomb oscillation peaks in these characteristics are associated with thermal currents through the QDs due to partial overlapping between the electrochemical potentials at small charging energies. To reduce these unimportant broadening effects from the characteristics and study pure electrostatic coupling we increase the charging energies of the QDs in the next simulations.

Fig. 2(d) demonstrates the grey scale plot of the simulated stability charge diagram at $4.2 \mathrm{~K}$ and $V_{\mathrm{d}}=2 \mathrm{mV}$ of $I_{\mathrm{ds}} \mathrm{vs} V_{\mathrm{g} 1}$ and $V_{\mathrm{g} 2}$ using Model ' $\mathrm{F}$ ' with charging energies of $40 \mathrm{meV}$ for QD1 and $55 \mathrm{meV}$ for QD2. For the comparison we used similar gate capacitances to Fig. 2(c). In contrast to Fig. 2(c) the broadening effects have disappeared in Fig. 2(d), due to the increase in the charging energies, and we only observe narrow oscillation peak lines (dark lines, Fig. 2(d)). The current is zero in the white regions (Fig. 2(d)) where the number of stable electrons in QD2 and QD1 are written between the brackets $((\mathrm{n} 2, \mathrm{n} 1)$ where $\mathrm{n} 2$ and $\mathrm{n} 1$ electrons are stable in QD2 and QD1 respectively). The dashed lines represent the boundaries for the regions of charge stability in QD1 and the peak lines form the boundaries for the regions of charge stability in QD2. Hexagonal regions of stable charges in the QDs are formed by the dashed and peak lines. Along a dashed line, the electrochemical potential of QD1 is aligned with the source-drain bias window and along a peak line the electrochemical potential in QD2 is aligned with the bias window. Theses potentials are related to each other via a linear relation [4] in $V_{\mathrm{g} 1}$ and $V_{\mathrm{g} 2}$ leading to the linear shift of the oscillation peaks as a function of $V_{\mathrm{g} 1}$ and $V_{\mathrm{g} 2}$. At the triple points (e.g. $p_{1}$ and $p_{2}$, Fig. 2(d)) the electrochemical potentials in the QDs are aligned with each other within the bias window allowing for sequential electron tunnelling (e.g. at $\mathrm{p}_{1}$, Fig. 2(d)) or hole tunnelling (e.g. at $\mathrm{p}_{2}$, Fig. 2(d)) between the source and drain through the QDs [4].

Along a peak line, electron tunnelling occurs between the source and drain through the electrochemical potential in QD2 which is aligned with the bias window. No current can occur at the dashed lines (Fig. 2(d)) because the electrochemical potentials in the QDs are misaligned and QD1 is disconnected from the drain. We investigated (data not shown) the effect of the coupling capacitance (capacitance 
of the tunnel barrier ' $t$ ', Fig. 2(b)) on the characteristics of Fig. 2(d). As the coupling capacitance increases from $\sim 0 \mathrm{aF}$ the splitting gap between adjacent triple points increases from $0 \mathrm{~V}$ and saturates at large coupling capacitance when the QDs coalesce to form an equivalent single QD.

Similar pattern of hexagonal regions to that of Fig. 2(d) is obtained using Model ' $\mathrm{S}$ ' and Model 'P' with similar gate capacitances and tunnel barrier capacitances ( $1 \mathrm{aF}$ each). However the stability diagrams for these models differ by the availability of the currents along the boundaries of the hexagonal regions. For example in Model ' $\mathrm{S}$ ' the current occurs only at the triple points (data not shown) and not along the boundaries of the hexagonal regions. This is because the QDs are coupled in series and no complete current path is formed between the source and drain across an individual QD.

Comparison between Figs. 2(d) and (c) shows that upon reducing the charging energies of the QDs the hexagonal regions of charge stability (white regions, Fig. 2(d)) evolve into narrow oscillation valley regions (white regions, Fig. 2(c)). For example the hexagonal regions $(-3,-3)$, $(-2,-2)$ and $(-1,-1)$ in Fig. 2(d) respectively correspond to the valley regions $V_{1}, V_{2}$, and $V_{3}$ in Fig. 2(c). Therefore the pattern formed by the narrow valleys may be associated with electrostatic coupling between QD1 and QD2 with small charging energies. This pattern is consistent with the experimental characteristics (Fig. 2(a)) of Device 1 which may similarly be associated with electrostatic coupling between two major nc-Si QDs in the point-contact channel with small charging energies.

Now we investigate the coherent coupling effects in Device 2. Fig. 3(a) shows the grey scale plot of the conductance $\left(\mathrm{d} I_{\mathrm{ds}} / \mathrm{d} V_{\mathrm{ds}}\right)$ of Device 2 at $4.2 \mathrm{~K}$ and $V_{\mathrm{ds}}=-2 \mathrm{mV}$ as a function of $V_{\mathrm{g} 1}$ and $V_{\mathrm{g} 2}$. The maximum conductance peak height is $0.3 \mathrm{nS}$ (dark regions). The characteristics were measured using standard Lock-in amplifier and current pre-amplifier set-up at excitation frequency and voltage of $13 \mathrm{~Hz}$ and $820 \mu \mathrm{V}$ respectively. Two peak lines (black dotted lines, Fig. 3(a)) intersect at the triple points ' $p_{1}$ ' and ' $\mathrm{p}_{2}$ ' forming partial patterns of hexagonal regions of charge stability in the coupled nc-Si QDs. In the vicinity of the triple points (region 'I', Fig. 3(a)), some peak structures have been observed. These structures are associated with coherent (without inelastic scattering) electron tunnelling between the source and drain across the delocalised electron wavefunctions over the coupled nc-Si QDs. We further investigated these structures by sweeping $V_{\mathrm{g} 1}$ horizontally (i.e. for constant $V_{\mathrm{g} 2}$ ) across region 'I' and found that they could be fitted to the sum of four Lorentzian peaks. The small peak structures gradually disappear from region 'I', and further along the dotted black lines (e.g. at the white arrow, Fig. 3(a)) only a single Lorentzian peak is seen.

Fig. 3(b) shows an example of the experimental characteristics (white squares) obtained by sweeping $V_{\mathrm{g} 1}$ across region 'I' (white dashed line, Fig. 3(a)) from $-2 \mathrm{~V}$ to $-0.5 \mathrm{~V}$ at $V_{\mathrm{g} 2}=-0.7 \mathrm{~V}$. The experimental curve is well fit-

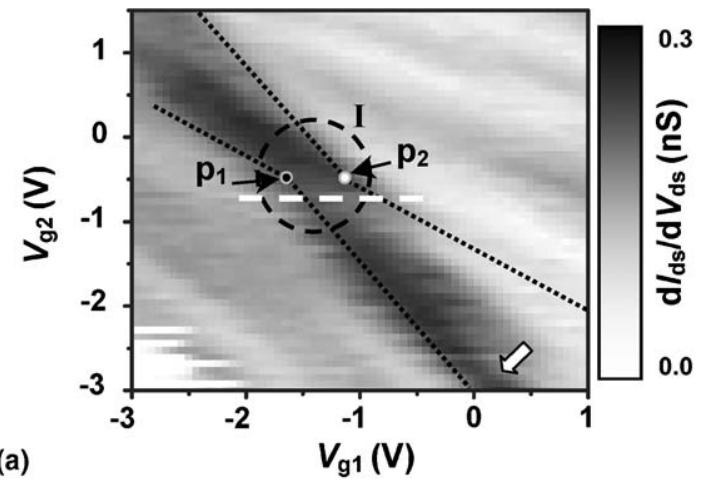

(a)

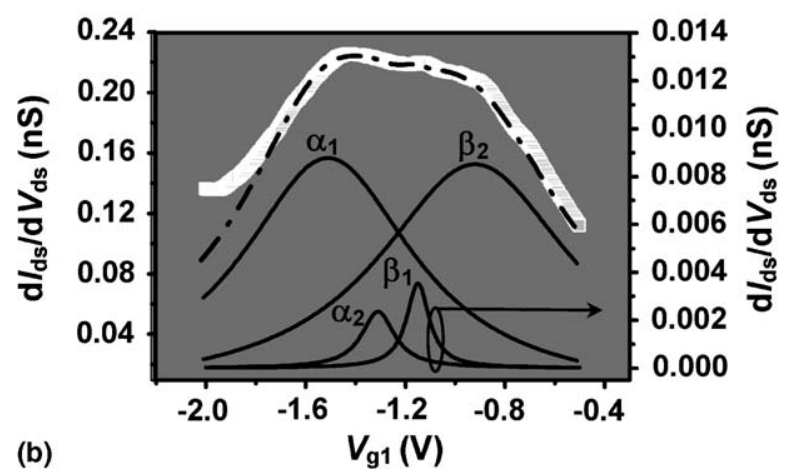

Fig. 3. (a) Experimental grey scale characteristics of the conductance, $\mathrm{d} I_{\mathrm{ds}} / \mathrm{d} V_{\mathrm{d}}$, in a point-contact transistor vs the gate voltages, $V_{\mathrm{g} 1}$ and $V_{\mathrm{g} 2}$, at source-drain bias $V_{\mathrm{ds}}=-2 \mathrm{mV}$ and at $T=4.2 \mathrm{~K}$. (b) White squares: drain-source conductance as a function of $V_{\mathrm{g} 1}$ at $V_{\mathrm{g} 2}=-0.7 \mathrm{~V}$, $V_{\mathrm{ds}}=-2 \mathrm{mV}$, and $T=4.2 \mathrm{~K}$ measured by sweeping $V_{\mathrm{g} 1}$ along the dashed white line in (b). The data can be fitted (dash dotted line) to the sum of four Lorentzian peaks $\alpha_{1}, \alpha_{2}, \beta_{1}$ and $\beta_{2}$.

ted (dash dotted line, Fig. 3(b)) to the sum of four Lorentzian peaks $\alpha_{1}, \alpha_{2}, \beta_{1}$, and $\beta_{2}$ (Fig. 3(b)). The Lorentzian peaks ' $\alpha_{1}$ and $\alpha_{2}$ ' and ' $\beta_{1}$, and $\beta_{2}$ ' are associated with electron tunnelling through the delocalised coherent levels near the triple points ' $p_{1}$ ' and ' $p_{2}$ ', respectively. The formation of these levels in our nc-Si point-contact transistors may be attributed to the coherent coupling across $\sim 1 \mathrm{~nm}$ thick grain boundary tunnel barrier between two nc-Si QDs with sizes smaller than or equal to the electron mean inelastic scattering path $(\sim 10 \mathrm{~nm}$ at $300 \mathrm{~K} \mathrm{[10]}$, and may be longer at $4.2 \mathrm{~K}$ ).

In conclusion, switching effects of the Coulomb oscillation peaks have been observed at $4.2 \mathrm{~K}$ in the current as a function of the gate voltages of oxidised and annealed nanometer scale nanocrystalline silicon (nc-Si) point-contact transistors. These effects were associated with electrostatic coupling between two major nc-Si grains in the point-contact channel. Monte Carlo single electron circuit simulations of models of two electrostatically coupled quantum dots (QDs) are consistent with the experimental results. An enhanced Coulomb oscillation peak switching (splitting) has been observed in the conductance as a function of the gate voltages of an oxidised-only device. The peak structures in the splitting region were well fitted to the sum of four Lorentzian peaks. These peaks were associated with coherent electron tunnelling (without inelastic scattering) across 
the delocalised electron wavefunctions over two coherently coupled nc-Si grains in the point-contact channel.

\section{Acknowledgments}

The authors would like to acknowledge Dr. T Kamiya of the Tokyo Institute of Tech. for helping with the fabrication, and Prof. H. Ahmed of the Microelectronics Research Centre, Cambridge University, for useful discussion and support.

\section{References}

[1] T. Ifuku, M. Otobe, A. Itoh, S. Oda, Jpn. J. Appl. Phys. 36 (1999) 4031.
[2] T. Kamiya, Z.A.K. Durrani, H. Ahmed, Appl. Phys. Lett. 81 (2002) 2388.

[3] Y.T. Tan, T. Kamiya, Z.A.K. Durrani, H. Ahmed, J. Appl. Phys. 94 (2003) 633 .

[4] W.G. van der Wiel, S. De Franceschi, J.M. Elzerman, T. Fujisawa, S. Tarucha, L.P. Kouwenhoven, Rev. Mod. Phys. 75 (2003) 1.

[5] M.A. Nielsen, I.L. Chuang, Quantum Computation and Quantum Information, Cambridge University Press, 2000.

[6] Z.A.K. Durrani, Physica E 17 (2003) 572.

[7] R.H. Blick, D. Pfannkuche, R.J. Haug, K. von Klitzing, K. Eberl, Phys. Rev. Lett. 80 (1998) 4032.

[8] A.J. Ferguson, D.G. Hasko, H. Ahmed, D.A. Williams, Appl. Phys. Lett. 82 (2003) 4492.

[9] P.A. Cain, H. Ahmed, D.A. Williams, J. Appl. Phys. 92 (2002) 346

[10] M.V. Fischetti, S.E. Laux, Phys. Rev. B 38 (1988) 9721. 